\title{
Psicología
}

Cartas al editor

\section{Un aporte del análisis bayesiano en la investigación psicológica}

\section{A contribution of Bayesian analysis in psychological Research}

\author{
Cristian Antony Ramos-Vera ${ }^{1 凶} \underline{\mathrm{ORCID}}$
}

${ }^{1}$ Psicologo. Área de investigación. Facultad de Ciencias de la Salud. Universidad Cesar Vallejo. Lima. Perú.

Fecha correspondencia:

Recibido: noviembre 1 de 2019.

Aceptado: junio 15 de 2021.

Forma de citar:

Ramos-Vera, C. (2021). Un aporte del análisis bayesiano en la investigación psicológica. Rev. CES Psico, 14(3), 191-194. https://dx.doi.org/10.21615/ cesp.5836

\section{Open access}

(C) Derecho de autor

Licencia creative commons

Ética de publicaciones

Revisión por pares

Gestión por Open Journal System DOI: $10.21615 /$ cesp.5836

ISSNe: 2011-3080

Publica con nosotros

\section{Sr. Editor}

En el estudio de Laderia y Grincenkov (2020), publicado en la presente revista, se reportaron asociaciones significativas, en una muestra brasileña formada por 25 pacientes con cáncer y sus cuidadores, en la puntuación de ansiedad (AN) y el dominio físico de la calidad de vida (DF) en ambos grupos, respectivamente, a través del test de significancia de la hipótesis nula (NHST, por sus siglas en ingles) de mayor uso en la investigación clínica. Se utilizó el coeficiente de correlación de Spearman, este análisis es debido en consideración del tamaño de muestra reducido y la distribución asimétrica de los datos (Laderia \& Grincenkov, 2020).

Los resultados fueron interpretados por el rechazo de la hipótesis nula a partir del nivel de significancia de 0.05 que permite asumir la hipótesis alterna de correlación a criterio del investigador (Leppink, O'Sullivan, \& Winston, 2017). Sin embargo, es importante la reevaluación estadística para reforzar la aceptación de las hipótesis frecuentistas en la investigación psicológica (Pek \& Van Zandt, 2019; Wagenmakers et al., 2018), por tanto, la presente carta tiene como objetivo presentar un ejemplo de replicación bayesiana. La estadística bayesiana permite contrastar hipótesis mediante probabilidades de credibilidad, con la ventaja de asegurar si la falta de significancia se debe a la insensibilidad de los datos o a la evidencia que respalda la falta de relación entre las variables, y de manera recíproca permite evaluar el nivel de evidencia probable de los valores significativos de las relaciones 
estadísticas y otros análisis basados en el nivel de significancia " $p<0.05$ ", siendo una alternativa metodológica que brinda mayor precisión a los análisis correlacionales (Pek \& Van Zandt, 2019; Ramos-Vera, 2021; Wagenmakers et al., 2018). Específicamente, el factor Bayes es el método que permite cuantificar el grado en que los datos apoyan tanto la hipótesis nula como la hipótesis alterna de la estadística frecuentista, cuyos valores se basan en el esquema de clasificación de Lee y Wagenmakers (2013): "débil", "moderado", "fuerte" y "muy fuerte" (ver Tabla 1).

Para la reevaluación bayesiana de los datos se tuvo en consideración el tamaño de las dos muestras ( $n=25, n=25)$ y los valores de correlación (Ly, Raj, Etz, Gronau, \& Wagenmakers, 2018; Quintana \& Williams, 2018). Las asociaciones significativas referidas en el primer párrafo entre AN y DF reportaron estimaciones de rho=-.457 y rho=-.475 en pacientes oncológicos y sus cuidadores respectivamente (Laderia \& Grincenkov, 2020).

Para la inferencia bayesiana se tuvo en cuenta dos interpretaciones del factor Bayes: FB10 (a favor de la hipótesis alternativa de significancia) y BF01 (a favor de la hipótesis nula) y el intervalo de credibilidad al 95\% con el fin de reevaluar la hipótesis de significancia de ambas correlaciones de AN-DF. Los resultados obtenidos del factor Bayes según el coeficiente de Kendall (ante la falta de normalidad de los datos) son BF10 $=33.88$ y BF01=0.03 e IC95\% [-.655 a -.152] y $B F 10=50.05$ y BF01=0.02 e IC95\% [-.670 a -.168], respectivamente. Este modelo bayesiano señaló una magnitud de evidencia muy fuerte en ambas hipótesis alternas de correlación entre AN-DF lo cual fortalece los resultados de Laderia y Grincenkov (2020), pues respaldan la evidencia a favor de la hipótesis de correlación 33 y 50 veces mayor a algún posible hallazgo de nulidad, por tanto, la hipótesis nula del factor Bayes que presenta una evidencia moderada se ve reducida en proporción, y no permite tomar en cuenta su interpretación. Asimismo, también se reporta el parámetro del factor Bayes máximo para determinar la estabilidad de los resultados, estos reportan valores muy similares, siendo mayores a 33 y 50 , respectivamente, avalando los resultados correlacionales significativos (Ly et al., 2018; Quintana \& Williams, 2018).

Por tanto, la inclusión del factor Bayes sería un gran aporte para el análisis probabilístico inferencial en el planteamiento de las hipótesis estadísticas, cuyo uso es inclusivo con diferentes pruebas estadísticas como el uso del estadístico t de Student que se evidencia en la literatura psicológica sudamericana reciente (Guillen \& Baños-Chaparro, 2021; Núñez, RamosVera, Serpa, \& Ogundokun, 2021). Además, permite la reevaluación de los resultados de investigaciones reales anteriores para determinar la certeza de sus conclusiones en análisis 
basados en NHST incluso cuando no se cuenta con acceso a los datos, reforzando también las investigaciones sistemáticas de metaanálisis, lo cual puede servir de base para futuros estudios en la revista que integren estos análisis como un aporte metodológico en el avance de la investigación psicológica (Pek \& Van Zandt, 2019).

Tabla 1. Valores de interpretación cuantificable del factor Bayes.

\begin{tabular}{lll}
$>30$ & Muy fuerte & Hipótesis alternativa \\
\hline $10+30$ & Fuerte & Hipótesis alternativa \\
\hline $3.1-10$ & Moderado & Hipótesis alternativa \\
\hline $1.1-3$ & Débil & Hipótesis alternativa \\
\hline 1 & 0 & No evidencia \\
\hline $0.3-0.9$ & Débil & Hipótesis nula \\
\hline $0.29-0.1$ & Moderado & Hipótesis nula \\
\hline $0.09-0.03$ & Fuerte & Hipótesis nula \\
\hline$<0.03$ & Muy fuerte & Hipótesis nula \\
\hline
\end{tabular}

Nota: Creación propia.

No hay conflicto de interés

Sin financiamiento

\section{Referencias}

Fuster, F. G., \& Baños-Chaparro, J. Análisis Bayesiano del compromiso académico en estudiantes de psicología: diferencias según sexo y edad. Revista de Investigación en Psicología, 24(1), 5-18. https://revistasinvestigacion.unmsm.edu.pe/index.php/psico/article/view/20210 Laderia, T., \& Grincenkov, F. (2020). Relação entre a Saúde Mental de pacientes com câncer avançado em quimioterapia paliativa e seus familiares cuidadores. Rev. CES Psico, 13(2), 1-17. http://doi.org/10.21615/cesp.13.2.1

Lee, M. D., \& Wagenmakers, E. J. (2014). Bayesian cognitive modeling: A practical course. Cambridge university press. Cambridge.

Leppink, J., O'sullivan, P., \& Winston, K. (2017). Evidence against vs. in favour of a null hypothesis. Perspectives on medical education, 6(2), 115-118. https://link.springer.com/article/10.1007/s40037-017-0332-6 
Ly, A., Raj, A., Etz, A., Marsman, M., Gronau, Q. F., \& Wagenmakers, E. J. (2018). Bayesian reanalyses from summary statistics: A guide for academic consumers. Advances in Methods and Practices in Psychological Science, 1(3), 367-374. https://journals.sagepub.com/doi/full/10.1177/2515245918779348

Núñez, C., Ramos-Vera, C.A., Serpa, A., \& Ogundokun, R.O. (2021). Adaptation of the Personality Type Inventory Based on Enneagram in Peruvian University Students of Health Sciences. Journal of Research in Medical and Dental Science, 9(5). https://doi.org/10.13140/RG.2.2.33090.30404

Pek, J., \& Van Zandt, T. (2019). Frequentist and Bayesian approaches to data analysis: Evaluation and estimation. Psychology Learning \& Teaching, 19(1), 21-35. https://doi.org/10.1177/1475725719874542

Quintana, D. S., \& Williams, D. R. (2018). Bayesian alternatives for common null-hypothesis significance tests in psychiatry: a non-technical guide using JASP. BMC psychiatry, 18, 178. https://doi.org/10.1186/s12888-018-1761-4

Ramos-Vera, C. A. (2021). The use of Bayes factor in clinical cardiology research. Revista Española de Cardiología, 74(7), 641-642. http://doi.org/10.1016/j.rec.2021.01.020

Wagenmakers, E. J., Love, J., Marsman, M., Jamil, T., Ly, A., Verhagen, J., ... Meerhoff, F. (2018). Bayesian inference for psychology. Part II: Example applications with JASP. Psychonomic bulletin \& review, 25(1), 58-76. https://doi.org/10.3758/s13423-017-1323-7 